\title{
Leyla Dakhli
}

\section{Napoléon a-t-il réveillé le monde arabe?}

Résumé: L'Expédition d'Egypte fait partie des dates canoniques de l'histoire contemporaine arabe. Elle est considérée comme une rupture majeure, faisant entrer cet espace dans la modernité. Cette chronologie est marquée par une conception très mécaniste de l'histoire, et par une vision des Lumières qui semble ne pas vouloir comparer Bonaparte avec les conquérants et les colonisateurs, en faisant même une exception, un faiseur de civilisation. Cette vision caricaturale, qui a alimenté l'orientalisme mais aussi les rêves d'émancipation arabes, est fortement discutée dès les premières décennies du XIX ${ }^{\mathrm{e}}$ siècle. L'objet de ce chapitre est de revenir sur ces discussions et sur ce qu'elles révèlent. Elles permettent de comprendre quelles circulations intellectuelles sont à l'œuvre dans cette première mondialisation méditerranéenne et ce qu'elles nous apprennent sur les universels imaginés de part et d'autre de cette petite mer.

Mots-clés: conquête/découverte; monde arabe; civilisation; langue(s); colonisation; nationalisme(s); Napoléon Bonaparte; Butrus al-Bustani; Ahmad Faris al-Shidiaq; Hassan al-Attar

La question peut paraître étrange, il faut le reconnaître. Mais elle est pourtant dérivée d'une des affirmations classiques de l'histoire contemporaine du monde arabe, qui date de l'expédition d'Égypte la renaissance de la région. Comme d'autres avant et après lui en d'autres espaces (Vigil 1990), cet Européen aurait donc découvert l'Égypte, l'aurait rendue à elle-même en lui faisant découvrir ses propres richesses, des pyramides aux obélisques, de la langue des tablettes à l'islam arabe. La critique de la notion de découverte a été faite ailleurs et fait partie d'un retour sur la modernité européenne. Le récit de la conquête qui nous est présenté aux pieds des pyramides est de courte durée, raconté sur le mode de la rencontre et de la révélation, et il n'est que l'un des tropes bien connu des livres d'histoire. Une des manières de renverser le stigmate serait - et a souvent été - de raconter le revers de la rencontre, et de montrer alors comment l'Égypte a conquis l'Europe, et même le monde en pénétrant son imaginaire, ses musées, ses salons, ses villes, suscitant même, après la publication des Voyages en Syrie et en Égypte de Volney (1799), le désir d'Égypte de Bonaparte (Humbert 1998); ou de raconter comment les Égyptiens ont ensuite rendu la politesse et à leur tour visité l'Europe,

Leyla Dakhli, CNRS/Centre Marc Bloch

2 Open Access. (C) 2021 Leyla Dakhli (c) BY-NC-ND This work is licensed under a Creative Commons Attribution-NonCommercial-No-Derivatives 4.0 License.

https://doi.org/10.1515/9783110691504-002 
raconté leurs découvertes et les merveilles de l'Occident. On peut ensuite raconter l'histoire de ce vice-roi d'Égypte, Mohammad Ali, qui voudra transformer les villes de son pays en Paris des Lumières, y mettre des opéras et des boulevards, des réverbères et des promenades. Enfin, on assistera au percement du Canal de Suez, micro-ouverture pour une nouvelle irrigation commerciale et culturelle de cette partie du monde qui, depuis des siècles, se trouvait contournée par le détroit de Magellan ${ }^{1}$.

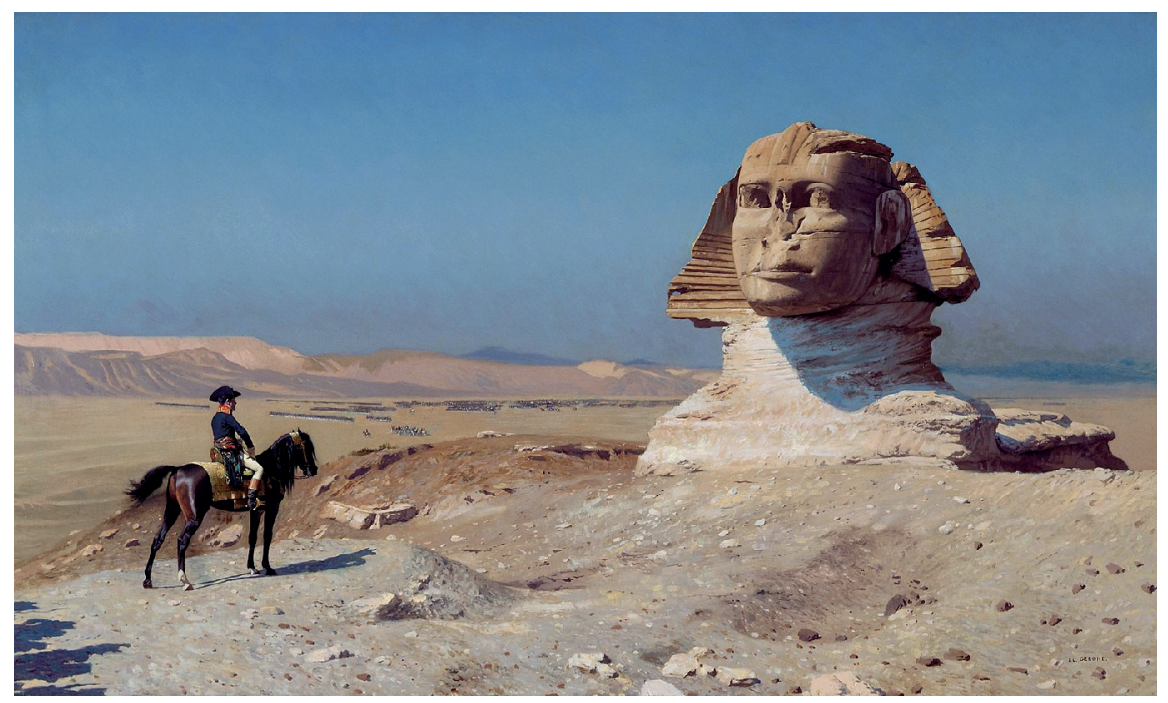

Fig. 1: « Bonaparte devant le Sphinx », 1886, par Jean-Léon Gérôme. Image: Wikimedia Commons. https://de.wikipedia.org/wiki/Datei:Bonaparte_ante_la_Esfinge,_por_JeanLéon_Gérôme.jpg (10.6.2020).

Mais c'est une vision qui reprend de toute façon le récit viriliste de la conquête et s'intéresse peu à la réalité des contacts et porosités. Et on peut aussi refuser de se laisser bercer ou berner par cette histoire rythmée par des gens de lettres et des aventuriers de papier, par des inventeurs et des déchiffreurs. S’il est passionnant de comprendre l'ampleur du rêve oriental du jeune Bonaparte qui, influencé par la lecture du Mahomet de Voltaire (2009 [1741]), se projette dans de nouvelles conquêtes sur des terres considérées comme à prendre et à réveiller après l'expédition italienne (Laurens 1989, 19-21), il pourrait être utile de voir si par hasard,

1 On peut renvoyer ici au chapitre de cet ouvrage sur l'inauguration du Canal de Suez; cf. l'article de Sarga Moussa. 
en tendant l'oreille, on ne peut pas comprendre autrement l'universalité de ce moment Bonaparte. Comme l'écrit Romain Bertrand, parler de la conquête, c'est toujours creuser un peu l'histoire de la violence en même temps que celle du remords. « Apprendre à exister dédoublé » devient dès lors l'une des figures de notre modernité (Bertrand 2015, 12).

Car lorsque le porteur de révolution et de lumières débarque en terre égyptienne, la Renaissance du monde arabe, la Nahda ${ }^{2}$, commence tout juste, et elle trouve dans cette guerre qui l'oppose à l'envahisseur européen une occasion de prendre la mesure de ce que la modernité représente, en matière de puissance de feu, mais aussi de développement intellectuel et scientifique. C'est à partir de ce constat que le projet moderniste égyptien se construit, en rupture avec l'Empire ottoman, en se cherchant d'autres supports et d'autres origines. Plus qu'une exportation des Lumières, on peut parler ici d'une secousse, voire d'une décharge électrique.

\section{Le contact}

On le voit, ce qui se joue d'abord autour de cette affaire, c'est une histoire de contact. On a pu donner à ce contact un sens, une direction. Les uns découvrant les autres, et leur apportant des choses (au choix : la civilisation, la science, les lumières, la révolution, les baïonnettes). C'est alors que la Renaissance arabe devient un mouvement nourri par ce contact, qui emprunte, voire adapte, ce que les européens lui font découvrir à son tour.

L'expédition napoléonienne est l'expérience de la sidération, ressentie notamment par les élites locales face au développement de la technique et de la science européennes. C'est ce qui est en partie lisible dans la célèbre chronique du cheikh d'Al-Azhar 'Abd al-Rahmân al-Jabarti (1753-1825), 'Aja'ib al-`athar fi al-tarâjim wal-`akhbar ${ }^{3}$. Cette vision détermine pour longtemps les dichotomies entre conservateurs et modernistes, séculiers et religieux. Al-Jabarti insiste par exemple sur sa fréquentation de la bibliothèque ouverte par les Français, ses échanges avec eux sur les questions scientifiques et sa découverte de l'orienta-

2 Le terme Nahda (essor, éveil, renaissance) désigne un mouvement intellectuel et culturel qui s'est développé à partir du début du XIX ${ }^{\mathrm{e}}$ siècle dans le monde arabe et musulman. Il désigne par extension et plus précisément sous le vocable devenu courant de 'asr al-`nahda une période d'effervescence liée notamment au développement de centres d'édition dans la région. Cette période s'étend du début du XIX ${ }^{\mathrm{e}}$ siècle à la fin de la Première Guerre mondiale. Cf. Dakhli, « Nahda ». 3 Traduction française de Joseph Cuoq sous le titre Journal d'un notable du Caire durant l'expédition française (1798-1801) ('Abd al-Rahmân al Jabarti 1979). 
lisme européen. La lecture de cette chronique montre bien la fascination pour les méthodes scientifiques qui sont utilisées par les Français. Néanmoins, les Égyptiens ont bien conscience d'être aussi des savants, et ils ont pour fonction d'enseigner à ces savants d'outre-mer.

Du contact, comme l'écrit Elliott Colla (2003), on connaît le plus souvent surtout la construction littéraire des Français qui décrivent comment les Égyptiens s'opposent à une mission venue leur apporter la Raison ou les libérer des despotes, selon les termes de Vivant Denon dans son Voyage dans la basse et la haute Égypte, mais aussi de Bonaparte lui-même;

Depuis trop longtemps les beys qui gouvernent l'Égypte insultent à la nation française, et couvrent ses négociants d'avanies: l'heure de leur châtiment est arrivée. Depuis trop longtemps ce ramassis d'esclaves achetés dans le Caucase et la Géorgie tyrannisent la plus belle partie du monde; mais Dieu, de qui dépend tout, a ordonné que leur empire finît.

Peuples de l'Égypte, on vous dira que je viens pour détruire votre religion; ne le croyez pas. Répondez que je viens vous restituer vos droits, punir les usurpateurs, et que je respecte, plus que les Mamelouks, Dieu, son prophète, et le Coran.

Dites-leur que tous les hommes sont égaux devant Dieu : la sagesse, les talents et les vertus mettent seuls de la différence entre eux.

Or quelle sagesse, quels talents, quelles vertus distinguent les Mamelouks pour qu'ils aient exclusivement tout ce qui rend la vie aimable et douce? Y a-t-il une belle terre? Elle appartient aux Mamelouks. Y a-t-il une belle esclave, un beau cheval, une belle maison? Cela appartient aux Mamelouks ${ }^{4}$.

Si l'Égypte est leur ferme, qu'ils montrent le bail que Dieu leur en a fait (Bonaparte, 1798, in Pujol 1845, 139-140).

Les « découvreurs de l’Égypte » y décrivent à foison l'ignorance et la confusion des « musulmans », subjugués par leurs maîtres eux-mêmes ignorants et cupides. Pourtant, ils sont aussi séduits par leur propre imaginaire oriental et les projections qu'il suscite sur la réalité lorsque Denon se met à " se rêver en Shahrayar » (Denon 1802, 105). Colla démontre que dans ce contact se joue aussi une forme d'homo-érotisation qui passe par le savoir, et qui concerne à la fois les textes de Denon sur les conteurs arabes et les textes de savants égyptiens comme Hassan al-Attar. Constater cette ambivalence, déjà présente dans une tradition soufie de désir pour le savoir, ne signifie évidemment pas relativiser la violence de la « rencontre coloniale » (Fauvelle-Aymar 1999; Bertrand 2001). Il s'agit simplement de comprendre peut-être un peu mieux les échanges qui ont lieu dans la découverte réciproque.

4 Pour une vision plus juste de l'origine de la dynastie des Mamelouks et leur œuvre, cf. Loiseau (2017). 
La poésie du savant d'al-Azhar Hassan al-Attar intitulée Maqâmat al-Fransis dit, dans la traduction de Colla:

He saw the affection in my eyes and spoke to me

With pearls of words, sweetly and effeminately

The beauty of seeing him became a metaphor that transforms lips into words

He pursued my mind with his gestures and, O my!

The Frenchmen have invaded everything, even my mind!

To consummate my love for him has become impossible

Because he has no pity for passion

I ask, « Shall we get together? » He replies, « Non! Non! »

I ask him, « Shall we leave each other? » He replies, « Si, Si! »

(Al-Attar, [1858], 96; traduit par Colla 2003, 1065)

Le jeu sur la notion d'invasion, qui pénètre jusqu'à l'esprit de ceux qui s'y soumettent, charrie un imaginaire érotique sans ambiguïté. Et le jeu sur les langues et les significations du refus disent évidemment un jeu du désir.

\section{Le réveil}

Qu'est-il resté de cet échange, reconnaissant la violence comme le désir mutuel, au moins dans la sphère du contact intellectuel, savant et littéraire? Sur ce contact, tel qu'il a été discuté par les historiens et les intellectuels égyptiens, on peut dire qu'il a été décliné sous la forme de la scansion régulière d'un "réveillez-vous, les Arabes ", qui repose sur un sentiment de perte, sur la conscience aiguë de se tenir sur des ruines. Il a été transformé en un discours critique qui fait de la modernité arabe un âge de l'imitation, de l'importation, de la dépendance, et du réveil, de la Nahda, le résultat de ce contact vécu comme une défaite. Le contact, en somme, installe un paradigme toujours prégnant pour la lecture historique des sociétés arabes, celui du retard. Ce que nous disent les envahisseurs, c'est que nous sommes faibles, et qu'il faut nous ressaisir, nous reconstituer pour ne plus être envahis. Bien plus, les envahisseurs doivent alors devenir nos modèles car ils ont les armes qu'il nous faut, et en premier lieu celles de la science et des techniques.

En cela, Napoléon et son expédition éclipsent d'autres paradigmes en construction, qui s'appuient sur d'autres types de rencontres, comme celle de l'intervention armée. La Renaissance arabe est alors provoquée par le choc d'une rencontre qui se poursuit au sein même des sociétés locales. Se transmet, en même temps que la conquête et ses ambiguïtés, la guerre et ses différentes formes. Les 
débuts de la mondialisation dans la région prennent alors la forme de conquêtes intérieures et de crises qui peuvent mener à la guerre civile. On le sait, les Mamelouks égyptiens partent bientôt à la conquête d'autres terres orientales, par-delà le Sinaï, et conquièrent la Palestine et la Syrie dans les premières décennies du $\mathrm{XIX}^{\mathrm{e}}$ siècle. La Renaissance arabe se construit aussi face à ce type de rencontre, et face au risque de la guerre civile. Dans le contexte de mondialisation et de transformation qui est celui du tournant du XIX ${ }^{\mathrm{e}}$ siècle, les sociétés du Moyen Orient et d'Afrique du Nord sont secouées et déstabilisées; elles se transforment et vivent des épisodes de violences interconfessionnelles qui accélèrent leurs transformations : émigrations, exode rural.

Ce que construisent aussi les auteurs et les autrices de ce temps, les acteurs et actrices de la Nahda, peut se comprendre comme une réponse à ces transformations, une réponse qui s'articule à la notion de patrie et trouve son ancrage dans la langue arabe. Plus que la question de l'emprunt, qui n'est en somme qu'un moyen, c'est la revivification, l'entretien systématique, à la manière d'un jardin, de la langue, qui est perçu comme une œuvre de civilisation.

L'un des auteurs majeurs de la Nahda, le Libanais ${ }^{5}$ Ahmad Faris al-Shidiyâq (1804-1887) considère dans les premières pages du merveilleux ouvrage autobiographique et picaresque qu'il rédige au milieu du XIX ${ }^{\mathrm{e}}$ siècle que les Arabes ont acquis leur civilisation par la langue, au contraire des Européens qui auraient acquis leur langue par la civilisation (al-Shidiaq, 1855; Traboulsi, 2016). Avec d'autres, il fait de ce constat le pilier majeur de son travail de revivification de la culture arabe. L'instrument majeur est la langue, la philologie, le travail sur les outillages conceptuels et sur les manières de dire le monde en arabe. On le voit, la question qui se pose alors est celle de l'origine et de la manière d'entrer dans la modernité.

De manière globale, le réveil s'opère alors que se déploie la mondialisation des conditions économiques et sociales par l'essor du capitalisme industriel. Dans les textes des auteurs du $\mathrm{XIX}^{\mathrm{e}}$ siècle, des références récurrentes sont faites à un âge d'or qu'il faudrait retrouver, mais aussi à un contexte qui se transforme sous leurs yeux de jour en jour. Le réveil est raconté comme faisant suite à une longue torpeur qui aurait saisi l'ensemble de la région après un temps d'effervescence et de rayonnement, ce que Rifaa al-Tahtâwî, dans l'exorde de son Or de Paris, appelle le « sommeil de l'incurie » (Al-Tahtâwî, 1834).

5 Les appartenances nationales ne sont alors pas fixées par des frontières, et la notion de Libanais est un peu anachronique. Shidiaq est un sujet ottoman, habitant des provinces arabes et du Mont-Liban, une région qui s’autonomise à l’issue des épisodes de violences confessionnelles de 1860. Shidiaq est par ailleurs un grand voyageur, il passe du temps en Égypte, en Afrique du Nord, en France et à Istanbul. 
La Nahda, qui se dit par la langue, est à la fois un grand mouvement de traduction et un mouvement de réappropriation des richesses de la langue arabe et de retrouvailles avec ses potentialités, comparable avec ce qu'on appelle en Islam l'ijtihad, cet effort de revivification et de développement, qui est exigé pour chacun des croyants et pour la collectivité bonne. Les textes de l'époque parlent souvent de l'emprunt (iqtibâs) comme un moyen de réveiller la culture et la science arabe et de combler le retard accumulé pendant des siècles d'inertie. Ceci devient par la suite un leitmotiv intellectuel, celui de la distance qui sépare les mondes civilisés sur ceux qui sont enfermés dans leur retard.

\section{La mondialisation et ses visages}

Pourtant, les racines endogènes de la Nahda ne se limitent pas à l'attachement des nahdaouis à la civilisation classique ou à l'héritage arabe, elles tiennent aussi à la continuité décelable en matière de développement économique et capitalistique, comme cela a été montré depuis longtemps (Gran 1998). Les liens qui existaient entre les rives de la Méditerranée, ou entre les mondes musulmans et chrétiens étaient fondés depuis des siècles sur le commerce et l'idée selon laquelle il y aurait eu un développement capitaliste européen complètement étanche est un peu saugrenue. C'est pourtant l'image qu'ont construite les orientalistes et celle que porte avec lui Napoléon, dressant le portrait d'un Empire ottoman inculte qui aurait détruit la splendeur de la civilisation égyptienne et aurait négligé le bonheur des habitants et leur épanouissement. À partir de cet ensemble de présupposés, voire de préjugés, peut se construire l'image de Bonaparte accueilli en sauveur et devenant le point de départ d'un sursaut, notamment pour les Égyptiens.

Pourtant, le lien entre les Égyptiens et les Français qui débarquent sur leurs rives est loin d'être idyllique. Comme l'écrivent Jens Hanssen et Hicham Safieddine, « à l'époque, l'armée de Napoléon fit face à un amusant mélange de réprobation et de ridicule ». Ils ajoutent: « Les chroniqueurs Abd al-Rahman al-Jabarti et Hassan al-Attar ne croyaient pas plus dans la rhétorique napoléonienne de libération de l'Égypte de l'oppression mamelouke que les Irakiens n'ont accueillis les soldats américains avec des fleurs en 2003 » (Hanssen et Safieddine 2019, 23).

Contrairement à ce que nous disent alors les voyageurs et les orientalistes, les intellectuels et savants arabes du début du XIX ${ }^{\mathrm{e}}$ siècle ne regardent pas que dans une direction, qui serait l'unique source de civilisation. Pour des raisons qui tiennent à des affinités religieuses, linguistiques, les mondes orientaux sont aussi connectés entre eux vers l'Est, et l'Asie comme la Perse sont des sources d'inspiration. Les circulations intellectuelles ne se limitent pas à un échange nord-sud, 
ou Europe-monde arabe, qui sera très largement construit dans la relation coloniale. On peut citer la figure célèbre de Jamal al-Dîn al-Afghâni (1838-1897): né dans une province afghane ou iranienne, formé entre l'Inde, l'Irak et l'Iran, émigré en Turquie, puis en Égypte et à Paris, il parle aussi bien le persan, le pachtou, l'ottoman que l'arabe. On peut aussi évoquer l'historien Mustafa Ali (15411600), musulman né à Gallipoli et mort à Djeddah. C’est sur ce socle, autant que sur le choc de la rencontre avec l'Occident, que se fonde l'entreprise de la Nahda. Dans le cadre d'un Empire à l'ancienne, où les provinces conservent leur autonomie, notamment sur le plan culturel, les élites intellectuelles, qu'elles soient artistiques, religieuses ou scientifiques, circulent et échangent. Elles partagent des références, se lisent dans différentes langues ${ }^{6}$. Elles se développent également en relation avec des lieux d'émigration, comme l'Amérique du Nord et du Sud. Par ailleurs, la présence de l'Europe dans le monde intellectuel arabe est multiforme : elle s’incarne aussi dans les écoles et universités missionnaires installées dans la région à partir du milieu du XIX ${ }^{\mathrm{e}}$ siècle. Cette présence européenne devient un des éléments de la culture locale. Ainsi la diffusion du darwinisme, un des pans importants de la pensée de la Nahda, se fait via le Syrian Protestant College de Beyrouth et suscite la polémique en son sein même. C'est à travers la polémique qui oppose un professeur, enseignant la théorie de Darwin, et la hiérarchie du College, qu'un groupe d'étudiants découvre les vertus émancipatrices des théories scientifiques et comment elles peuvent s'opposer à l'ordre missionnaire. Ces histoires sont bien connues, et elles nous transportent vers d'autres types de contacts et de rencontres.

Dans le contexte d'une première mondialisation, les mouvements qui suivent les guerres et les conquêtes napoléoniennes ont charrié des revendications nationales et d'émancipation. S'il est bien clair que ce langage n'est pas celui qui a été entendu lors de l'expédition de 1798, les effets des guerres révolutionnaires, des printemps des peuples sont eux directement ressentis dans l'Empire ottoman puisqu'ils se déroulent à ses portes, et en son sein. La Nahda peut alors être lue comme un mouvement des nationalités arabe, et un mouvement d'émancipation sociale.

Ne se limitant pas à un mouvement proto nationaliste arabe, elle embrasse la question de l'émancipation de multiples manières, en la réinterprétant à l'aune $\mathrm{du}$ contexte moyen oriental. La focalisation des acteurs de la Nahda sur les questions de langue et d'identité est incontestable. Elle a certainement permis de for-

\footnotetext{
6 Sur ce point, voir la revision opérée par le grand historien Albert Hourani $(1991,128)$ : « It now seems to me to have been wrong in laying too much emphasis upon ideas which were taken from Europe, and not enough upon what was retained, even if in a changed form, from an older tradition. » Hourani in International Journal of Middle Eastern Studies, 1991, p. 128.
} 
ger les concepts clés du nationalisme arabe. Mais cette dimension linguistique et d'auto-désignation comme « Arabes » s'opère dans le cadre de l'Empire ottoman, et, majoritairement, dans une grande fidélité à la Porte. À travers cette recherche, elle touche à des domaines comme le rationalisme ${ }^{7}$, la sécularisation des sociétés - notamment par la critique du système des millets ottomans, la place des plus faibles et d'une classe ouvrière cosmopolite (et souvent européenne) de plus en plus visible dans les grandes villes et les ports (Khuri-Makdisi 2010 ; Turiano 2016, 337-349), ou encore la place des femmes.

Le texte que fait paraître Butrus al-Bustani, « Le Clairon de la Syrie » [Nafîr Suriyya] pendant les affrontements confessionnels de 1860 dans la montagne libanaise est un bel exemple de la réflexion complexe qui s'opère autour de la notion de watan (patrie, heimat). La définition de la nation n'est pas simple dans une région qui découvre dès les années 1840 puis plus violemment encore en 1860 les affres de la guerre civile, al-harb al-ahliyya, que l'on pourrait traduire plutôt par « guerre domestique ». On y trouve une réflexion forte sur le vivre ensemble, à partir de l'observation d'une crise locale. Ces idées font partie du bagage que se constituent les penseurs et penseuses arabes dans le contact avec les transformations de la modernité. Comme l'Empire austro-hongrois, l'Empire ottoman vit cette période comme un moment de dislocation des équilibres et d'affirmation de volontés d'émancipation tout autant que d'invention de nouvelles solidarités, dont la patrie ou la communauté est l'une des formes.

Le mouvement nahdaoui est d'emblée un mouvement politique, dans la mesure où il prend position sur l'avenir de l'ordre politique impérial, sur ses caractéristiques, sur ses réformes, dans la mesure aussi où cette prise de parole s'opère à partir de la crise des équilibres sociaux, économiques et culturels antérieurs, crise qui ne se résume pas à l'attirance - réelle - pour les idéaux émancipateurs de la Révolution française, ou à la soumission - chèrement obtenue aux nouveaux ordres de domination impériale du monde. On sait que la Première Guerre mondiale sera l'occasion d'imposer de ces nouveaux systèmes de domination jusque dans le Proche et Moyen Orient, après les premières conquêtes coloniales en Afrique du Nord.

Pendant que les canons prennent position, les intellectuel-le·s forgent d'autres armes pour décrire et désigner le monde qui change, puis pour gouverner, juger. Butrus al-Bustani, par-delà les pamphlets politiques, publie des dictionnaires (Muhît al-`muhît - L'Océan, [1869-1870]), des lexiques de la langue nouvelle (Lûghat al-`jarâ’id, La langue de la presse, 1901), une encyclopédie de la langue actuelle (Dâ'irat al-`ma'ârif, entreprise menée avec son fils Salîm, et restée inache-

7 Cf. La controverse entre Renan et Afghani, cf. Renan (2005). 
vée). Il tente de pallier les insuffisances qu'il a lui-même déplorées et de mettre la langue à niveau afin d'éviter sa subordination. Pour lui comme pour les autres, l'instrument majeur de cette résistance passe par la culture.

Il ne nous appartient pas ici de dire si l'on peut parler de défaite ou de victoire face aux forces de la conquête et du contact. Ce que Napoléon était venu chercher, il l'a emporté avec lui, ceci nous le savons. Ce qu'il prétendait être venu apporter, il est plus difficile de savoir si cela a été absorbé, détourné, et s'il n’a pas apporté bien d'autres choses dont il ne se serait pas vanté, à commencer par la guerre. Cette guerre n'est pas seulement celle, meurtrière, des canons. Elle est aussi celle que l'on dirait aujourd'hui des civilisations. Ce que dit Bonaparte en prenant pied en Égypte, ce qu'il affirme, c'est qu'il y a nous et les autres, un autre radical que l'on peut conquérir, que l'on peut même aimer, mais qui reste radicalement autre. L'un des représentants les plus fameux de l'historiographie issue de cette conception est certainement l'ottomaniste ultra-conservateur Bernard Lewis, notamment via son (bel) ouvrage Comment l'Islam a découvertl'Europe (Lewis 1984). Le livre est une mine d'informations, mais il contribue à fixer l'idée d'un contact entre deux entités fixes, qu'il qualifiera par la suite de civilisations qui entrent en choc. Les frontières poreuses entre ces civilisations, dont témoignent notamment les sources qu'il cite abondamment dans ses ouvrages, ne sont rien en regard de l'irréductibilité de deux ensembles, Islam et Chrétienté, que Napoléon lui-même semble mimer lors de son arrivée en Égypte. Napoléon n’a pas réveillé le monde arabe, il l'a simplement fait exister comme un monde radicalement autre. En Égypte, il a pris ce qui lui appartenait: obélisques, statues, pierre de Rosette, jusqu'aux Dieux pharaoniques qui sont devenus les berceaux d'une humanité commune qui siège dans les musées d'Occident. Et il a observé, soumis et fait le projet de transformer une autre humanité contemporaine, considérée quant à elle comme radicalement autre. Aucun de ces deux legs ne fut approprié par les intellectuel-le·s arabes, à part peut-être cette barrière érigée entre les mondes, qui leur fût d'autant plus visible que bientôt elle s'arma et les poussa à s'armer à leur tour. Dans ce jeu de miroir réside une bonne part des remords de la conquête, et des malentendus de ce qu'on appelle modernité.

\section{Références bibliographiques}

Al-Bustani, Butrus. Muhit al-Muhit 2 vols. Beirut: Librairie du Liban, 1979.

Al-Jabarti, 'Abd al-Rahmân. Journal d'un notable du Caire durant l'expédition française (17981801). Trad. et notes Joseph Cuoq. Paris: Albin Michel, 1979.

Al-Attar, Hasan. Al-Maqama fi-l-fransis. Bulaq, [1858]. 
Bertrand, Romain. «La rencontre coloniale, une affaire de mœurs? L'aristocratie de Java face au pouvoir hollandais à la fin du XIXe siècle». Genèses XLIII.2 (2001): 32-52.

-. Le Long remords de la conquête. Paris: Seuil, 2015.

Colla, Elliott. «"Non, Non! Si, Si!”: Commemorating the French Occupation of Egypt (17981801)». MLN CXVIII.4 (2003): 1043-1069.

Dakhli, Leyla. «Nahda». Notice pour H. Touati (dir.). Encyclopédie de l'humanisme méditerranéen (2014). http://www.encyclopedie-humanisme.com/?Nahda (10 septembre 2020).

Denon, Vivant. Voyage dans la basse et la haute Égypte. Le Caire: Institut Français d'Archéologie Orientale du Caire, 1989 [1802].

Fauvelle-Aymar, François-Xavier. «La rencontre coloniale. Regards sur le quotidien». Politique africaine LXXIV.2 (1999): 105-112.

Gran, Peter. Islamic Roots of Capitalism. Egypt 1760-1840. New York: Syracuse University Press, 1998.

Hanssen, Jens et Hicham Safieddine. The Clarion of Syria. Berkeley: University of California Press, 2019.

Hourani, Albert. «How should we write the history of the Middle East?». International Journal of Middle East Studies XXIII.2 (1991): 128.

Humbert, Jean-Marcel. Rêve d'Égypte. Catalogue d'exposition. Paris, 1998.

Khuri-Makdisi, Ilham. The Eastern Mediterranean and the Making of Global Radicalism, 18601914. Berkeley: University of California Press, 2010.

Laurens, Henry. L'Expédition d'Égypte (1798-1801). Paris: Armand Colin, 1989.

Lewis, Bernard. Comment l'Islam a découvert l'Europe. Trad. Annick Pélissier. Paris: Gallimard, 2005.

Loiseau, Julien. Les Mamelouks (XIII $-X V l^{e}$ siècle). Une expérience du pouvoir dans l'Islam médiéval. Paris: Seuil, 2017.

Pujol, Auguste. Fuvres choisies de Napoléon. Mises en ordre et précédées d'une étude littéraire. Paris: Belin-Leprieur éditeur, 1845.

Renan, Ernest. L'Islam et la science. Montpellier: L’Archange Minotaure, 2005.

Al-Shidiaq, Ahmad Faris. La Jambe sur la jambe [Al-Sâq 'alâ al-Sâq]. Trad. (incomplète) René R. Khawam. Paris: Phébus, 1991 [1855]. [Une traduction complète en 2 volumes a été réalisée en langue anglaise par Humphrey Davies: Leg over Leg. New York: NYU Press, 2013].

Al-Tahtawi, Rifâ‘a Rif'at. L'Or de Paris. Relation de voyage, 1826-1831 [Takhlis al-lbriz fi talkhis baris]. Trad. Anouar Louca. Paris: Sindbad, 1988 [1834].

Traboulsi, Fawaz. «Ahmad Faris Al-Shidyaq (1804-1887)». Arabic Thought beyond the Liberal Age: Towards an Intellectual History of the Nahda. Éds Jens Hanssen et Max Weiss. Cambridge: Cambridge University Press, 2016, 175-186.

Turiano, Annalaura. «Le consul, le missionnaire et le migrant. Contrôler et encadrer la main d'œuvre italienne à Alexandrie à la fin du XIX' siècle». Étudier en liberté les mondes méditerranéens. Mélanges offerts à Robert llbert. Éds Leyla Dakhli et Vincent Lemire. Paris: Publications de la Sorbonne, 2016, 337-349.

Vigil, Ralph H. «Spanish exploration and the Great Plains in the age of discovery: myth and reality». Great Plains Quarterly 10 (1990): 3-17.

Volney, Constantine-François de Chassebœuf, Comte de. Voyage en Syrie et en Égypte, Pendant les années 1783, 1784 et 1785. Paris: Dugour et Durand, 1799.

Voltaire. Le Fanatisme ou Mahomet le prophète. Paris: Fayard (« Milles et une nuits »), 2009 [1741]. 
